\title{
Mortalidade por homicídios em linha de fronteira no Paraná, Brasil
}

\author{
Homicide mortality in border regions in the State of Paraná, Brazil
}

Vinícius Denepotti Nogueira (https://orcid.org/0000-0001-6998-9222) ${ }^{1}$

Ludmila Mourão Xavier-Gomes (https://orcid.org/0000-0001-6442-5719) ${ }^{1}$

Thiago Luis de Andrade Barbosa (https://orcid.org/0000-0002-6985-9548) ${ }^{1}$
${ }^{1}$ Instituto Latino-

Americano de Ciências da Vida e da Natureza,

Universidade Federal

da Integração Latino-

Americana. Av. Tarquínio Joslin dos Santos 1000

Bairro Polo Universitário.

85870-901 Foz do Iguaçu

PR Brasil.

vinicius.nogueira@

hotmail.com.br

\begin{abstract}
A time series analysis was conducted to identify trends in homicide mortality in border regions in the State of Paraná, Brazil between 2002 and 2012. Homicide mortality rates were analyzed by sex, age group, region (border regions, non-border regions, and the state as a whole), and type of assault. Trend analysis was performed using polynomial regression. The findings showed that mortality rates were higher in the border regions; however the growth in homicide rates was greater in the non-border regions and in the state as a whole, with these states showing an upward trend in homicide rates $(p<0.001)$ and border regions showing a downward trend, although the latter was not significant. Mortality rates were higher among men across all regions and highest in the 20 to 29 age group. The findings regarding homicide rates in border regions provide compelling evidence of the urgent need for intersectoral prevention policies targeting the most affected groups. Key words Homicide, Mortality, Border areas, External causes
\end{abstract}

Resumo Estudo de série temporal que objetivou analisar a tendência dos homicídios em residentes da linha de fronteira no estado do Paraná, Brasil, no período de 2002 a 2012. Os óbitos por homicídios foram analisados de acordo com variáveis de idade, sexo, região de residência e meio usado para realizar a agressão. A análise de dados empregada foi a regressão polinomial. Os resultados mostram que as taxas de mortalidade são maiores em linha de fronteira ao longo da série, porém houve maior incremento desses indicadores na região não fronteiriça e no Paraná com tendência crescente $(p<0,001)$. Os homicídios na linha de fronteira apresentam tendência decrescente, porém não significativa. A mortalidade foi maior entre homens e no grupamento etário de 20 a $29 \mathrm{em}$ todas as regiões investigadas. A magnitude dos homicídios na linha de fronteira é um alerta para sugerir a elaboração de políticas públicas intersetoriais de prevenção voltados para os grupos acometidos. Palavras-chave Homicídio, Mortalidade, Áreas de fronteira, Causas externas 


\section{Introdução}

No Brasil, os homicídios representam as maiores taxas de óbito entre as causas externas. Em 2015, foram 59.080 óbitos, número equivalente a taxa de 28,9 mortes por 100 mil habitantes, o que representa mais de $10 \%$ dos homicídios de todo o mundo. Essa causa de óbito implica em mudanças na dinâmica social, econômica, demográfica e de saúde das populações ${ }^{1}$.

A violência ocasiona grandes perdas para $o$ país, com impacto no desenvolvimento econômico e indicadores sociais e reflete de maneira direta nos gastos em saúde, sensação de insegurança e bem-estar social ${ }^{2}$. Violência e ferimentos têm destaque na mortalidade e morbidade no Brasil desde a década de 1980, com grande representatividade entre os homens jovens ${ }^{3}$. Analisar os óbitos por homicídios contribui para a compreensão de suas tendências e torna possível o aprimoramento de intervenções de políticas públicas de saúde a fim de reduzir indicadores e aprimorar os serviços prestados a comunidade ${ }^{4}$.

As regiões de fronteira tradicionalmente têm sido consideradas território marginal, periférico e com déficits de integração socioeconômica, contudo verificam-se mudanças e desenvolvimento regional ao longo do tempo. Esse processo destaca-se pelo impacto direto na saúde enquanto componente primário e hábil para integração entre a sociedade e a população fronteiriça. Com a dinâmica econômica dessa região, o enfretamento à violência, inclusão social, ações de educação e a segurança nacional refletem de forma direta para o sucesso das políticas públicas de saúde ${ }^{5}$.

Especificamente, o Paraná, localizado na região sul brasileira, faz fronteira com três estados do Brasil (Mato Grosso do Sul, São Paulo e Santa Catarina), ao leste encontra-se o Oceano Atlântico, e mais dois países da América Latina ao oeste, sendo eles Argentina e Paraguai. O contorno fronteiriço deste estado representa $2.458 \mathrm{~km}$, com $447 \mathrm{~km}$ de região de fronteira com os países da América do Sul. A maioria das fronteiras no estado do Paraná são feitas por meio naturais, como rios e os divisores de águas, e o restante por fronteiras artificiais. Entre as fronteiras do estado, destaca-se a do Rio Paraná que separa o município brasileiro de Foz do Iguaçu do município paraguaio de Ciudad del Este, e o Rio Iguaçu que separa Foz do Iguaçu do município argentino Puerto Iguazú ${ }^{6}$.

Há singularidades da linha de fronteira internacional com territórios com limites políticos bem definidos, dinâmica econômica própria, livre fluxo de pessoas, relações históricas com questões culturais e sociais? ${ }^{7}$. Isso contribui para a diversificação dos óbitos em linha de fronteira, como no estado do Paraná, principalmente dos homicídios e acidentes de transporte. Sabese que são poucos os estudos que verificaram a tendência dos homicídios em linha de fronteira em todo o mundo, assim a literatura brasileira e internacional apresenta carência de estudos nessa linha de investigação. Por representar um ambiente de contato permanente e acentuado, a linha de fronteira internacional demarca países com realidades distintas. Conhecer a magnitude da violência na fronteira sul do país demonstra a necessidade de pesquisas mais detalhadas e a aplicação de políticas públicas que visem combater as taxas de violência e superar outras carências dessa população. Nesse contexto este estudo teve por objetivo investigar a tendência dos homicídios em residentes de linha de fronteira no estado do Paraná.

\section{Métodos}

Trata-se de estudo de série temporal sobre a tendência da mortalidade por homicídios no estado do Paraná, região sul do Brasil, construído a partir de dados oficiais e secundários do Sistema de Informações sobre Mortalidade (SIM) do Ministério da Saúde.

Os dados foram coletados do Departamento de Informática do Sistema Único de Saúde (Datasus). Foram considerados os óbitos por residência ocorridos no período entre 2002 e 2012. Tomou-se como base a Classificação Internacional de Doenças da 10a revisão (CID-10) para registro dos óbitos por agressões (X-85 a Y-09). Os dados demográficos foram obtidos a partir de dados censitários e estimativas populacionais do Instituto Brasileiro de Geografia e Estatística (IBGE). Para comparar os homicídios por localidade, considerou-se a divisão do estado em linha de fronteira (17 municípios - Barracão, Bom Jesus do Sul, Capanema, Entre Rios do Oeste, Foz do Iguaçu, Guaíra, Itaipulândia, Marechal Cândido Rondon, Mercedes, Pato Bragado, Pérola d'Oeste, Planalto, Pranchita, Santa Helena, Santo Antônio do Sudoeste, São Miguel do Iguaçu e Serranópolis do Iguaçu) e região não fronteiriça (382 municípios) conforme Figura 1. Os casos com informações ignoradas não foram incluídos na descrição dos dados.

Os óbitos por homicídios foram analisados de acordo com variáveis de idade (anos), sexo 


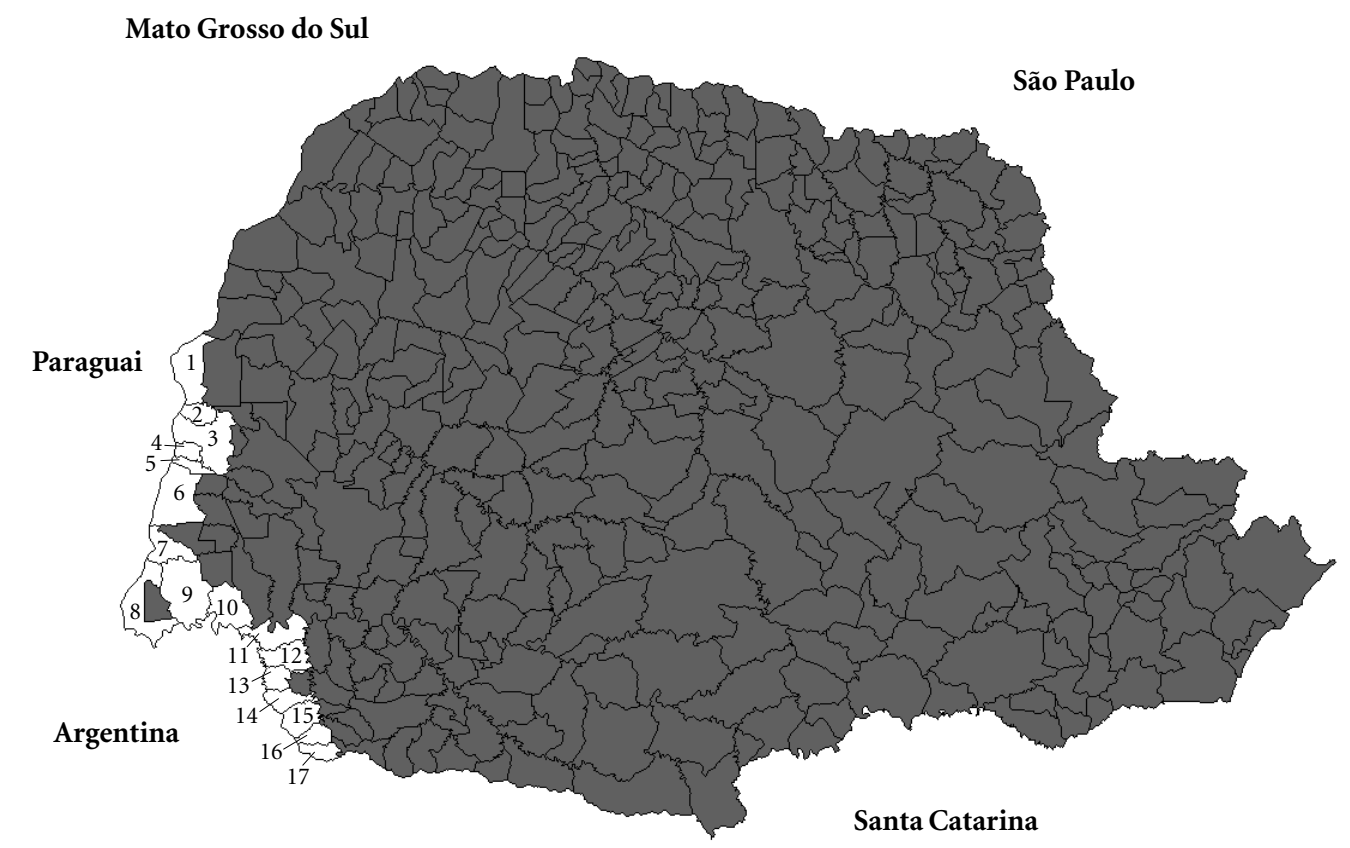

Municípios de linha de fronteira

1. Guaíra

2. Mercedes

3. Marechal Cândido Rondon

4. Pato Bragado

5. Entre Rios do Oeste

6. Santa Helena

7. Itaipulândia

8. Foz do Iguaçu

9. São Miguel do Iguaçu

10. Serranópolis do Iguaçu

11. Capanema

12. Planalto

13. Pérola d'Oeste

14. Pranchita

Santa Catarina

15. Santo Antônio do Sudoeste

16. Bom Jesus do Sul

17. Barracão

Figura 1. Mapa do estado do Paraná (Brasil), com destaque para linha de fronteira com Argentina e Paraguai.

Fonte: SIM/MS/DATASUS.

(masculino/feminino), região de residência (linha de fronteira/região não fronteiriça/Paraná) e meio usado para realizar a agressão. Nesta variável, os homicídios foram agrupados da seguinte forma: X93 a X95 agressão por armas de fogo, X99 agressão por objeto cortante ou penetrante, Y00 agressão por meio de um objeto contundente e os demais agrupados em outros meios de agressão.

Foram calculadas as taxas de mortalidade pela razão do número de óbitos ocorridos no ano, considerando a população em risco do mesmo período por 100 mil habitantes. Para efeito de comparação das taxas ao longo da série histórica, realizou-se a padronização pelo método direto por idade. Nesse método de ajuste, utilizase uma população padrão no sentido de afastar a possibilidade de resultados encontrados serem diferentes daquelas existentes na distribuição etária da população. Ademais, é empregado para comparar duas ou mais populações com diferenças em suas estruturas etárias ou uma mesma 
população em períodos distintos. Para realizar esse método delimita-se uma população padrão torna-se a referência para os demais anos da série temporal ${ }^{8}$. A população padrão considerada foi a do ano de 2010 do IBGE, correspondente ao ano do último censo.

As taxas de mortalidade por homicídios foram analisadas por sexo, e faixa etária (10 a 19 anos, 20 a 29 anos, 30 a 39 anos, 40 a 49 anos, 50 a 59 anos e 60 anos ou mais) em linha de fronteira e região não fronteiriça do Paraná. A análise de tendência foi realizada por meio do modelo de regressão polinominal'.

As taxas de mortalidade padronizadas por homicídio foram empregadas como variáveis $(\mathrm{Y}$ - dependente), enquanto os anos sequenciais foram utilizados como variáveis independentes ( $\mathrm{X}$ - independente). Isso foi centralizado no ponto médio da série para evitar a autocorrelação entre os termos na equação de regressão (X - 2007). As parcelas de dispersão de variáveis independentes foram utilizadas para Função que melhor expressou o relacionamento entre eles. Subsequentemente, a primeira ordem $\left(Y=\beta_{0}+\beta_{1} x\right)$, segunda ordem $\left(\mathrm{Y}=\beta_{0}+\beta_{1} \mathrm{x}+\beta_{2} \mathrm{x}^{2}\right)$, terceira ordem ( $\mathrm{Y}$ $\left.=\beta_{0}+\beta_{1} \mathrm{x}+\beta_{2} \mathrm{x}^{2}+\beta_{3} \mathrm{x}^{3}\right)$ e exponencial $\left(\mathrm{Y}=\mathrm{e}^{\beta 0+}\right.$ ß1x) foram testados, em que $\beta_{0}$ é o coeficiente médio para o período e $\beta_{1}$ é a taxa média anual. A escolha do modelo baseou-se nos seguintes critérios: melhor função de acordo com o diagrama de dispersão, melhor ajustado (normalidade de erros e homoscedasticidade), maior significância estatística (ANOVA p-valor) e maior coeficiente de determinação $\left(R^{2}\right)$. Nos casos de modelos estatisticamente semelhantes, o mais simples foi selecionado. O nível de significância adotado foi 5\%.

O software Microsoft Excel 2007 foi utilizado para construção de indicadores, padronização e preparação de tabelas e gráficos. Para análise da tendência da mortalidade por homicídios, utilizou-se o software estatístico Bioestat 5.0 da Universidade Federal do Pará (UFPA) e Tabwin for Windows do Datasus.

\section{Resultados}

No período analisado, o estado do Paraná contabilizou 688.626 óbitos, sendo que 96.039 foram devidos às causas externas, representando $13,9 \%$ do total. Dessas, 34.200 correspondem a óbitos por homicídios totalizando $35,6 \%$ das mortes devido a causas externas. A Tabela 1 mostra a evolução das taxas de mortalidade por homicídios nas regiões estudadas e no estado do Paraná, segundo sexo. A magnitude das taxas na linha de fronteira é maior que da região não fronteiriça até o ano de 2007. Após esse período observa-se inversão dessa situação na qual as taxas de homicídios dessa última tornam-se mais elevadas. $\mathrm{Na}$ linha de fronteira, a taxa de homicídio passou de 71,2/100 mil habitantes, em 2002, para 60,0/100 mil habitantes em 2012, o que representa decréscimo de $15,7 \%$ do risco. Por outro lado, na região não fronteiriça e no estado do Paraná houve aumento importante de $38,0 \%$ ao longo dos anos observados. Destaca-se o comportamento dos indicadores em linha de fronteira no período de 2007 a 2009 com diminuição de 32,5\%, passando de 77,4/100 mil habitantes para 52,2/100 mil habitantes. Nos anos seguintes, houve declínio com oscilações.

Constatou-se que a mortalidade masculina foi maior em todas as regiões analisadas. Na linha de fronteira ocorreu redução de $16,2 \%$ das taxas de mortalidade masculina quando comparado o ano de 2002 e 2012. No estado do Paraná ocorreu aumento de $37,6 \%$, no mesmo período, com maior incremento dos indicadores no intervalo de 2002 a 2008. Em relação à mortalidade feminina, observou-se padrão diferente ao do sexo masculino em relação a linha de fronteira, pois houve aumento de $3,7 \%$ das taxas ao passo que no estado houve aumento de $42,5 \%$, no mesmo público. Em relação à proporção média masculino/feminino constatada foi 13:1 para linha de fronteira e 11:1 para região não fronteiriça e Paraná.

A Figura 2 revela a evolução das taxas de homicídios por sexo, segundo faixa etária e região, nos anos 2002, 2007 e 2012. O grupamento etário de 20 a 29 anos apresentou as maiores taxas de mortalidade nas regiões investigadas em ambos os sexos. Nesse grupo, constata-se aumento de $56,7 \%$ em região não fronteiriça, $43,4 \%$ no Paraná e redução de $25,8 \%$ na linha de fronteira quando comparado o primeiro e último ano da série histórica. Em indivíduos com 60 anos ou mais, ocorreu diminuição das taxas de homicídios em ambos os sexos, todavia com queda mais acentuada no sexo masculino quando comparado com a faixa etária anterior (50 a 59 anos).

Em relação ao meio utilizado para perpetrar o homicídio (Tabela 2), houve predomínio do uso de armas de fogo em todas as regiões. $\mathrm{Na}$ linha de fronteira, essa causa representava mais de três quartos do total de homicídios, mesmo com o declínio observado ao longo do período estudado. Nessa região, o uso de objeto cortante ou penetrante aumentou ao passo que houve decréscimo dos óbitos causados pelo uso das armas 
Tabela 1. Taxas de mortalidade por homicídios (por 100.000 hab.), segundo sexo e região. Paraná, Brasil, 20022012.

\begin{tabular}{lccccccccc}
\hline & \multicolumn{3}{c}{ Linha de fronteira } & \multicolumn{3}{c}{ Região não fronteiriça } & \multicolumn{3}{c}{ Paraná } \\
\cline { 2 - 10 } Ano & Masculino & Feminino & Total & Masculino & Feminino & Total & Masculino & Feminino & Total \\
\cline { 2 - 10 } & Taxa & Taxa & Taxa & Taxa & Taxa & Taxa & Taxa & Taxa & Taxa \\
\hline 2002 & 136,9 & 8,1 & 71,2 & 45,2 & 4,5 & 45,2 & 49,9 & 4,7 & 26,8 \\
2003 & 114,6 & 10,0 & 61,3 & 52,6 & 4,9 & 52,6 & 55,7 & 5,1 & 29,9 \\
2004 & 144,0 & 12,0 & 76,7 & 56,2 & 5,4 & 56,2 & 60,7 & 5,7 & 32,6 \\
2005 & 140,6 & 9,9 & 73,8 & 58,6 & 5,0 & 58,6 & 62,8 & 5,2 & 33,3 \\
2006 & 150,7 & 11,2 & 79,4 & 60,2 & 5,2 & 60,2 & 64,9 & 5,5 & 34,5 \\
2007 & 145,4 & 11,8 & 77,4 & 58,7 & 4,6 & 58,7 & 63,2 & 5,0 & 33,5 \\
2008 & 115,9 & 10,0 & 62,1 & 65,8 & 6,0 & 65,8 & 68,4 & 6,2 & 36,7 \\
2009 & 97,9 & 7,9 & 52,2 & 70,7 & 6,9 & 70,7 & 72,2 & 6,9 & 38,9 \\
2010 & 113,6 & 9,8 & 60,2 & 69,9 & 7,0 & 69,9 & 71,9 & 7,1 & 38,8 \\
2011 & 99,5 & 7,0 & 51,9 & 65,1 & 5,8 & 65,1 & 66,7 & 5,9 & 35,6 \\
2012 & 114,6 & 8,4 & 60,0 & 66,5 & 6,6 & 66,5 & 68,7 & 6,7 & 37,0 \\
\hline
\end{tabular}

Fonte: SIM/MS/Datasus.

de fogo. No Paraná a proporção do uso de armas de fogo manteve-se alta, com elevação entre os anos analisados, enquanto a agressão por objeto cortante ou penetrante obteve uma redução. As agressões por meio não especificado reduziram no final do período analisado no estado e região não fronteiriça.

A Tabela 3 representa a análise de dados por meio de estimação de curvas de regressão para as taxas de mortalidade total e segundo sexo para as regiões estudadas. Nesse estudo, constatou-se no estado do Paraná tendência crescente quadrática para o total $\left(\mathrm{R}^{2}=0,762\right.$; $(\mathrm{p}<0,001)$ e sexo masculino $\left(\mathrm{R}^{2}=0,874 ; \mathrm{p}<0,001\right)$ e linear para o sexo feminino $\left(\mathrm{R}^{2}=0,587 ; \mathrm{p}=0,001\right)$. Para a região não fronteiriça, houve tendência crescente linear $\left(\mathrm{R}^{2}=0,762 ;(\mathrm{p}<0,001)\right.$, quadrática para o sexo masculino $\left(\mathrm{R}^{2}=0,874 ; \mathrm{p}<0,001\right)$ e exponencial para o sexo feminino $\left(\mathrm{R}^{2}=0,587 ; \mathrm{p}=0,001\right)$. Os homicídios na linha de fronteira apresentaram altas taxas com tendência decrescente, sendo exponencial para taxa total $(\mathrm{R} 2=0,319 ; \mathrm{p}=0,041)$, exponencial para o sexo masculino $(\mathrm{R} 2=0,307$; $\mathrm{p}=0,045)$ e cúbica para o sexo feminino $(\mathrm{R} 2=$ $0,525 ; \mathrm{p}=0,042)$.

\section{Discussão}

Os achados deste estudo permitiram focalizar a situação dos homicídios em duas regiões do estado do Paraná (fronteira e não fronteira) com a detecção da existência de situações distintas, evidenciando elementos que podem contribuir para a formulação de propostas de intervenção mais efetivas. No período analisado, os resultados revelaram elevada magnitude das taxas de homicídio na linha de fronteira internacional, principalmente para o sexo masculino.

Investigação realizada com as regionais de saúde do Paraná, no período de 1979 a 2005, revelou uma elevação do risco dos homicídios na maioria das regionais, com destaque para as de fronteira como a de Foz do Iguaçu ${ }^{10}$. O presente estudo apontou, no entanto, tendência decrescente das taxas de mortalidade em linha de fronteira e crescente dos óbitos na região não fronteiriça e no Paraná ao longo da série temporal. Esse fato corrobora com a questão de o estado apresentar as maiores taxas de homicídios da região sul do país ${ }^{1,10}$. Pesquisa nacional que mapeou a violência no país apontou, após o ano de 2010, realidade distinta para os estados nos quais se observaram decréscimo representativos das taxas de homicídios: Espírito Santo (-27,6\%), Paraná $(-23,4 \%)$ e Alagoas $(-21,8 \%)^{1}$. Essa diminuição pode ter sido atribuída à implementação do Estatuto do Desarmamento, campanhas de recolhimento de armas de fogo, maior repressão policial com crescimento das apreensões e prisões, e projetos de inclusão social para adolescentes ${ }^{1,11}$.

Vale ressaltar que, mesmo com o declínio observado no Paraná, a linha de fronteira apresentou as maiores taxas de homicídio, ao considerar o conjunto de municípios dessa região. Historicamente, a faixa de fronteira brasileira configura- 

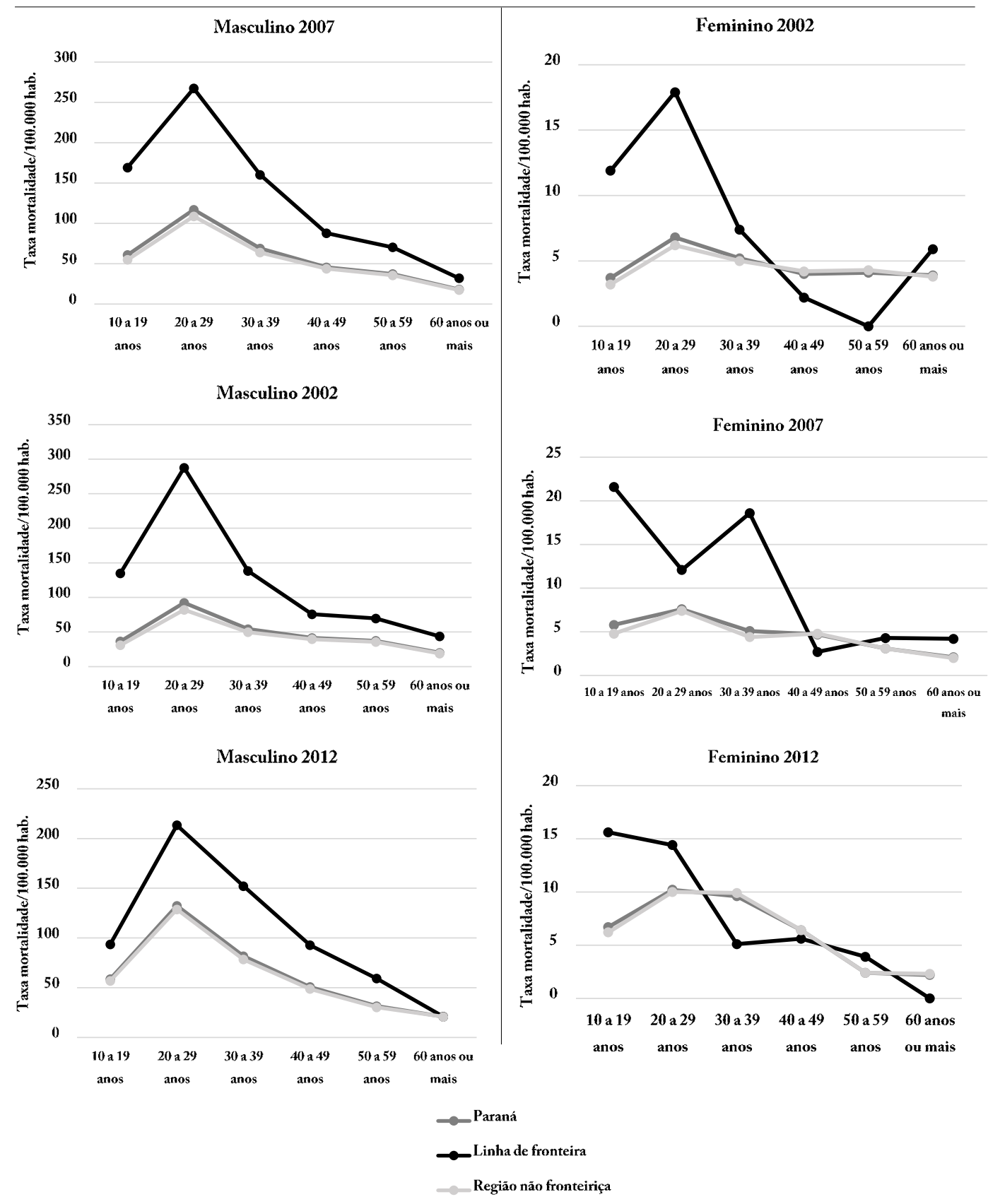

Figura 2. Taxas de mortalidade por homicídios por idade e sexo, segundo região, Paraná, Brasil, 2002-2012.

Fonte: SIM/MS/Datasus.

se como pouco desenvolvida, abandonada pelo Estado e marcada por dificuldades de acesso a bens e serviços públicos de saúde, educação e segurança pública ${ }^{12}$. Nesse contexto, os municípios fronteiriços do Paraná compartilham situação semelhante em carência de recursos em ambos os lados da fronteira, com fluxos transfrontei- riços constantes e/ou presença de populações vulneráveis, o que pode refletir diretamente nos indicadores de violência.

A linha de fronteira internacional do Paraná é marcada por intenso trânsito de pessoas e veículos haja vista as atividades econômicas baseadas no cultivo de grãos (soja, cana-de-açúcar, milho), 
Tabela 2. Distribuição percentual dos homicídios por meio utilizado segundo região. Paraná, Brasil, 2002-2012.

\begin{tabular}{|c|c|c|c|c|c|c|c|c|c|c|c|}
\hline \multicolumn{12}{|c|}{ Paraná } \\
\hline \multirow{3}{*}{ Causa do Óbito } & \multicolumn{11}{|c|}{ Ano } \\
\hline & 2002 & 2003 & 2004 & 2005 & 2006 & 2007 & 2008 & 2009 & 2010 & 2011 & 2012 \\
\hline & $\%$ & $\%$ & $\%$ & $\%$ & $\%$ & $\%$ & $\%$ & $\%$ & $\%$ & $\%$ & $\%$ \\
\hline $\begin{array}{l}\text { Agressão por armas de fogo } \\
\text { (X93 a X95) }\end{array}$ & 67,4 & 69,8 & 68,6 & 68,7 & 72,5 & 74,0 & 74,4 & 72,9 & 74,2 & 71,8 & 70,9 \\
\hline $\begin{array}{l}\text { Agressão objeto cortante ou } \\
\text { penetrante (X99) }\end{array}$ & 18,3 & 16,9 & 18,2 & 17,4 & 16,4 & 15,1 & 14,3 & 15,2 & 13,8 & 16,0 & 16,3 \\
\hline $\begin{array}{l}\text { Agressão p/meio de objeto } \\
\text { contundente (Y00) }\end{array}$ & 6,8 & 6,0 & 5,5 & 6,0 & 5,0 & 5,4 & 5,0 & 5,5 & 5,2 & 5,6 & 6,6 \\
\hline $\begin{array}{l}\text { Meio não especificado (X85 a } \\
\text { X92; X96 a X98; Y01 a Y09) }\end{array}$ & 7,5 & 7,3 & 7,7 & 7,8 & 6,1 & 5,5 & 6,4 & 6,5 & 6,9 & 6,6 & 6,2 \\
\hline Total & 100,0 & 100,0 & 100,0 & 100,0 & 100,0 & 100,0 & 100,0 & 100,0 & 100,0 & 100,0 & 100,0 \\
\hline \multicolumn{12}{|c|}{ Região não fronteiriça } \\
\hline \multirow{3}{*}{ Causa do Óbito } & \multicolumn{11}{|c|}{ Ano } \\
\hline & 2002 & 2003 & 2004 & 2005 & 2006 & 2007 & 2008 & 2009 & 2010 & 2011 & 2012 \\
\hline & $\%$ & $\%$ & $\%$ & $\%$ & $\%$ & $\%$ & $\%$ & $\%$ & $\%$ & $\%$ & $\%$ \\
\hline $\begin{array}{l}\text { Agressão por armas de fogo } \\
\text { (X93 a X95) }\end{array}$ & 64,1 & 68,3 & 65,9 & 66,2 & 70,1 & 72,1 & 73,0 & 72,1 & 73,5 & 71,1 & 70,1 \\
\hline $\begin{array}{l}\text { Agressão objeto cortante ou } \\
\text { penetrante (X99) }\end{array}$ & 19,9 & 17,4 & 19,6 & 18,7 & 17,7 & 16,1 & 14,9 & 15,4 & 13,9 & 16,4 & 16,5 \\
\hline $\begin{array}{l}\text { Agressão p/meio de objeto } \\
\text { contundente (Y00) }\end{array}$ & 7,5 & 6,3 & 6,0 & 6,4 & 5,4 & 5,7 & 5,2 & 5,7 & 5,3 & 5,7 & 6,9 \\
\hline $\begin{array}{l}\text { Meio não especificado (X85 a } \\
\text { X92; X96 a X98; Y01 a Y09) }\end{array}$ & 8,6 & 8,1 & 8,5 & 8,7 & 6,8 & 6,1 & 6,9 & 6,8 & 7,3 & 6,8 & 6,5 \\
\hline Total & 100,0 & 100,0 & 100,0 & 100,0 & 100,0 & 100,0 & 100,0 & 100,0 & 100,0 & 100,0 & 100,0 \\
\hline \multicolumn{12}{|c|}{ Linha de fronteira } \\
\hline & & & & & & Ano & & & & & \\
\hline \multirow[t]{2}{*}{ Causa do Óbito } & 2002 & 2003 & 2004 & 2005 & 2006 & 2007 & 2008 & 2009 & 2010 & 2011 & 2012 \\
\hline & $\%$ & $\%$ & $\%$ & $\%$ & $\%$ & $\%$ & $\%$ & $\%$ & $\%$ & $\%$ & $\%$ \\
\hline $\begin{array}{l}\text { Agressão por armas de fogo } \\
\text { (X93 a X95) }\end{array}$ & 88,4 & 82,8 & 88,3 & 88,0 & 89,6 & 87,6 & 88,3 & 83,4 & 82,4 & 81,0 & 81,2 \\
\hline $\begin{array}{l}\text { Agressão objeto cortante ou } \\
\text { penetrante (X99) }\end{array}$ & 8,3 & 12,6 & 7,5 & 7,5 & 6,8 & 8,0 & 7,7 & 11,9 & 11,6 & 10,6 & 13,2 \\
\hline $\begin{array}{l}\text { Agressão p/meio de objeto } \\
\text { contundente (Y00) }\end{array}$ & 2,3 & 3,4 & 2,1 & 3,0 & 2,5 & 3,6 & 2,7 & 2,8 & 4,0 & 4,6 & 2,4 \\
\hline $\begin{array}{l}\text { Meio não especificado (X85 a } \\
\text { X92; X96 a X98; Y01 a Y09) }\end{array}$ & 1,0 & 1,1 & 2,1 & 1,5 & 1,1 & 0,8 & 1,3 & 2,0 & 2,0 & 3,7 & 3,2 \\
\hline Total & 100,0 & 100,0 & 100,0 & 100,0 & 100,0 & 100,0 & 100,0 & 100,0 & 100,0 & 100,0 & 100,0 \\
\hline
\end{tabular}

escoamento de produção através de "porto seco" de outras regiões brasileiras e do Paraguai e Argentina e indústrias alimentícias regionais. Esse contexto pode contribuir para elucidar a magnitude desses indicadores que pode ser devida, ainda, a aglomerações urbanas, maior proporção de jovens de 15 a 24 anos, nível educacional e ao índice de Gini (indicador de desigualdades sociais), como consequência da desigualdade social que afasta a população dos processos produtivos e dos bens de consumo, além de questões migratórias próprias ${ }^{13-15}$.

Outra questão relacionada aos homicídios consiste no fato de a linha de fronteira constituir importante aporte de tráfico de drogas. Com a dissipação do uso da cocaína nos anos 1980, o Brasil inseriu-se na rota do tráfico, por estar situado em localização próxima de países produtores 
Tabela 3. Análise das tendências de mortalidade por homicídios (total e por sexo), segundo regiões geográficas. Paraná, Brasil, 2002-2012.

\begin{tabular}{llrrc}
\hline \multicolumn{1}{c}{ Taxa de Mortalidade } & \multicolumn{1}{c}{ Modelo $^{*}$} & $\mathbf{R}^{\mathbf{2}^{* *}}$ & \multicolumn{1}{c}{$\mathbf{p}^{* * *}$} & Tendência \\
\hline Paraná Total & $\mathrm{Y}=-0,1539 \mathrm{x}^{2}+2,8106 \mathrm{x}+24,534$ & 0,865 & $<0,001$ & Crescente \\
Masculino & $\mathrm{Y}=-0,304 \mathrm{x}^{2}+5,4135 \mathrm{x}+45,599$ & 0,895 & $<0,001$ & Crescente \\
Feminino & $\mathrm{Y}=0,1937 \mathrm{x}+4,6652$ & 0,601 & 0,003 & Crescente \\
Linha de fronteira Total & $\mathrm{Y}=77,728 \mathrm{e}^{-0,029 \mathrm{x}}$ & 0,319 & 0,041 & Decrescente \\
Masculino & $\mathrm{Y}=146,75 \mathrm{e}^{-0,029 \mathrm{x}}$ & 0,307 & 0,045 & Decrescente \\
Feminino & $\mathrm{Y}=0,0264 \mathrm{x}^{3}-0,577 \mathrm{x}^{2}+3,4098 \mathrm{x}+5,2828$ & 0,525 & 0,042 & Decrescente \\
Região não fronteiriça Total & $\mathrm{Y}=1,1243 \mathrm{x}+25,893$ & 0,762 & $<0,001$ & Crescente \\
Masculino & $\mathrm{Y}=-0,2795 \mathrm{x}^{2}+5,4227 \mathrm{x}+41,188$ & 0,874 & $<0,001$ & Crescente \\
Feminino & $\mathrm{Y}=4,4227 \mathrm{e}^{0,0382 \mathrm{x}}$ & 0,587 & 0,004 & Crescente \\
\hline
\end{tabular}

*Modelo: $\mathrm{Y}=$ Taxa de mortalidade por acidente de trânsito por 100.000 habitantes (global e por categoria de vítima); $\mathrm{X}=$ ano 2007; ${ }^{* *} \mathrm{R}^{2}$ = coeficiente de determinação; ${ }^{* * *} \mathrm{p}$-valor do Teste $\mathrm{F}$.

Fonte: SIM/MS/Datasus.

da droga e contato estreito com países da Europa e América do Norte. Tal situação favoreceu a expansão da violência, crime organizado, comércio ilegal de armas e atividades ilícitas, constantemente, em linha de fronteira ${ }^{11}$.

As regiões de fronteira são marcadas ainda por iniquidades, amplo tráfico de pessoas, questões diplomáticas e territórios contínuos entre as nações, apresentando dificuldades de melhor monitoramento. Esses aspectos podem ajudar a compreender a situação dos óbitos por homicídios nos municípios da linha de fronteira, no que se refere às diferenças sociais, econômicas e demográficas que repercute nas desigualdades socioeconômicas e de acesso a serviços básicos de saúde e educação. Vale ressaltar que a maior parte dos homicídios ocorre, principalmente, nos municípios fronteiriços com porte populacional de 200 a 300 mil habitantes que se destacam por maior violência, atividade de contrabando de eletrônicos, cigarros, armas, tráfico de drogas para o Brasil ${ }^{11}$.

Os resultados deste estudo relevam a importância da mortalidade masculina por homicídios entre os adultos jovens (20 a 39 anos) nas regiões estudadas do Paraná, com maiores taxas de homicídio na linha de fronteira quando comparadas com as do sexo feminino. Investigação realizada em Maceió ${ }^{16}$, Alagoas, demonstrou que $94,8 \%$ das vítimas de homicídios eram do sexo masculino e 66,2\%, jovens na faixa etária de 15 a 29 anos, corroborando com este estudo. Perfil semelhante é registrado em estudos conduzidos no Chile, Colômbia e África do Sul ${ }^{17-19}$.

$\mathrm{Na}$ Colômbia encontrou-se a razão homem/ mulher de 10:1, com maior taxa de mortalidade na faixa etária de adulto jovem ${ }^{18}$. No Brasil ${ }^{20}$, as agressões constituem 10,0\% da carga total de doenças, sendo que a relação de anos potenciais de vida perdidos foi de 4,8 homem/mulher. Neste estudo, a razão na linha de fronteira foi de 13:1, superior à do estado do Paraná e a do estudo anterior. Em relação a esse resultado, os homens nos municípios de linha de fronteira podem estar apresentando estilo de vida mais exposto aos riscos de serem vítimas de incidentes violentos, além de provocar maior perda de anos de vida. $\mathrm{O}$ envolvimento no tráfico de drogas e o contrabando de mercadorias associados a comportamentos viris, autocráticos e desafiadores que afligem a identidade de autoafirmação podem ter como efeito a criação de um círculo de violência entre os jovens. Esse comportamento possui a probabilidade de estender-se à vida adulta, conservando os riscos de gerar e sofrer agressão ${ }^{21,22}$. Esse fato merece atenção das autoridades, pois atinge um grupo de ampla produção econômica e inserção social nos municípios de fronteira, acarretando maiores gastos com o sistema de saúde, decorrentes de lesões provocadas por agressões ${ }^{4}$.

A mortalidade feminina possui elevação na mesma faixa etária do sexo masculino, principalmente na linha da fronteira. Esse comportamento pode estar atrelado a violência contra a mulher nas relações de gênero e, também, a fatores culturais envolvendo Brasil, Paraguai e Argentina. Nessas realidades ocorrem a dominação masculina que pode estar ligada a falta de proteção contra as diferentes formas de violência domésticas e crimes passionais. Esses atos quando não resultam em óbito, possuem aspectos negativos na esfera econômica, coletiva e sobre o núcleo familiar. No Brasil foi sancionada a Lei no 11.340 
(Lei Maria da Penha), que visa reprimir, principalmente a violência doméstica e familiar contra a mulher ${ }^{23}$. Porém, a efetividade dessa Lei temse mostrado restrita, pois ainda não conseguiu cumprir integralmente sua proposta. A elevação de óbitos femininos por homicídio no presente estudo merece atenção singular dos gestores públicos, para que a Lei seja cumprida e a impunidade não se torne fato corriqueiro na linha de fronteira, para que impacte de maneira positiva na redução de agressão e óbito entre mulheres vítimas de violência. Sugere-se novas investigações que possam elucidar melhor a situação de violência contra a mulher em área de fronteira.

A menor variação da taxa de mortalidade de mulheres idosas quando comparada com a faixa etária anterior (50-59 anos), revela que esse último grupo se encontra mais vulnerável a óbitos por homicídio. Dados semelhantes quanto a essa faixa etária de acometimento são encontrados em outros estudos ${ }^{24,25}$. Entretanto, vale pontuar que essa situação de mortalidade pode estar atrelada ao efeito do pequeno número de pessoas nesta faixa etária de modo que qualquer alteração no número de óbitos afeta muito a taxa, seja para cima ou para baixo. No Chile, no período de 2000-2012, o grupo de 60 anos ou mais é o terceiro mais acometido por homicídios ficando atrás apenas dos grupos de 25-39 e 40-59 anos ${ }^{17}$. Estudo realizado no município de Foz do Iguaçu ${ }^{24}$, Paraná, localizado na tríplice fronteira com Argentina e Paraguai, 2002-2010, relatou redução de $25,7 \%$ da taxa de mortalidade feminina por homicídios na faixa etária de 50 a 59 anos. Esse município, em particular, apesar de pertencer a linha de fronteira e ser o maior em porte populacional, a retração apresentada não impactou na elevação dessas taxas considerando toda região. Isso pode implicar que municípios menores estão apresentando maior risco de homicídios entre as mulheres, o que implica novas investigações. Outra implicação, como relatado anteriormente, seria que o efeito do pequeno número de mulheres nesta faixa etária pode elevar ou diminuir as taxas facilmente, de acordo com o número de óbitos apresentados.

Em relação às causas específicas, é importante destacar, na presente investigação, os homicídios causados por arma de fogo nas regiões analisadas compõem percentual superior em relação a outras formas de agressão, corroborando com achados de estudos realizados no Brasil, Colômbia e África do Sul ${ }^{13,18,19}$. A arma de fogo tem sido o meio mais utilizado para o homicídio, seguida pela arma branca ${ }^{16,20,26}$ o que pode indicar a intenção de letalidade da agressão, diminuindo as chances de sobrevivência da vítima e aumentando a eficácia do ato.

No Brasil, em 2013, entrou em vigor a Lei $n^{\circ}$ 10.826 que dispõe sobre o registro, posse e comercialização de armas de fogo e munição. Assim, foram lançadas campanhas de entrega de armas, ou políticas buy-pack, em que o estado compra a arma de fogo por um valor pré-definido do cidadão que a entrega de maneira voluntária ${ }^{27}$. Estudo realizado no Paraná apontou que a campanha de desarmamento voluntário não produziu resultados significativos em curto prazo, pois a campanha representou pequena escala em comparação ao estoque de armas do país, criminosos ou pessoas que tendem a atos violentos não entregarem as armas ${ }^{28}$. Apesar desse contexto, este trabalho mostrou que a proporção de homicídios por armas de fogo permaneceu elevada durante toda série histórica, sendo acima de 70\% em todas as áreas analisadas com destaque para linha de fronteira com $81,2 \%$ dos homicídios por arma de fogo. A linha de fronteira se destaca por ser uma área de grande movimentação do tráfico de armas e drogas, com destaque para Foz do Iguaçu, mesmo com ações das forças de polícia e do Estado para coibir essa situação ${ }^{24}$.

Outro fato que se destaca neste estudo é o aumento do uso de arma branca como meio utilizado para homicídio na linha de fronteira. Apesar dessa elevação, a arma de fogo continua sendo o principal meio perpetrado para cometer homicídio. A arma branca é um meio de homicídio bastante utilizado pelo sexo masculino, principalmente pelos adultos jovens e adolescentes ${ }^{29}$. O aumento das proporções de homicídios por armas brancas nos municípios de linha de fronteira pode estar relacionado a diminuição do contrabando de armas de fogo nessa região, favorecendo homicídios por outro meio de fácil acesso.

Com relação ao estudo, existem limitações que devem ser ponderadas na avaliação dos resultados. Ao se utilizar dados secundários do SIM, as notificações insuficientes e variações da especificidade da informação, como no caso de óbitos por causas indeterminadas, podem interferir diretamente na qualidade e quantidade dos dados. Associado a isso o menor percentual de dados processados no SIM provenientes de fontes oficiais e problemas relacionados na emissão da Declaração de Óbito (DO) e subnotificações ${ }^{30}$. A subnotificação dos óbitos pode dificultar a construção de indicadores que representem melhor a realidade da linha de fronteira. Contudo, vale ressaltar que a qualidade do preenchimento da declaração de óbito por causas externas tem melhorado em todo país. As 
menores taxas de óbitos por homicídios no sexo feminino não devem subestimar a magnitude da mortalidade nesse grupo, pois possui alta relevância por se tratar de um reflexo da essência sociocultural e de gênero do processo de violência. Informações sobre a mortalidade feminina podem ser um indício para serviços que atendam esse grupo em situação de risco.

Conclui-se que houve diminuição das taxas de homicídios em linha de fronteira ao longo da série temporal, mas permanece elevado em relação a região não fronteiriça e no estado do Paraná. Isso merece esforços cada vez maiores para reduzir essa causa externa em uma região vulnerável e de alta dinâmica econômica, social, política e demográfica. Destaca-se o sexo mascu- lino e adulto jovem como a grande faixa etária acometida que representam grande parte da população produtiva do país. Achados desse estudo permitem identificar grupos de maior risco e as tendências temporais, contribuindo assim, para sugerir a elaboração de políticas públicas intersetoriais de prevenção voltados para os grupos acometidos. Configura um desafio aos profissionais de saúde, gestores públicos e autoridades políticas para a inclusão do tema nas prioridades de saúde nos municípios de linha de fronteira. Portanto, conhecer a magnitude, determinação e a tendência dos óbitos por homicídios tende a auxiliar a implementação de ações efetivas na redução e prevenção desses fatos.

\section{Colaboradores}

VD Nogueira trabalhou na concepção, delineamento, coleta de dados, análise e interpretação dos dados, redação do artigo e aprovação da versão a ser publicada. LMX Gomes trabalhou na concepção, delineamento, revisão crítica do artigo e aprovação da versão a ser publicada. TLA Barbosa trabalhou na concepção, delineamento, coleta de dados, análise e interpretação dos dados, redação do artigo e aprovação da versão a ser publicada.

\section{Agradecimentos}

À Universidade Federal da Integração Latino-Americana (UNILA) pelo apoio financeiro. 


\section{Referências}

1. Cerqueira D, Lima RS, Bueno S, Valencia LI, Hanashiro O, Machado PHG, Lima AS. Atlas da violência 2017. Rio de Janeiro: IPEA; 2017.

2. Tavares R, Catalan VD, Romano PMM, Melo EM. Homicídios e vulnerabilidade social. Cien Saude Colet 2016; 21(3):923-934.

3. Reichenheim ME, Souza ER, Moraes CL, Mello Jorge MHP, Silva CMFP, Minayo MCS. Violência e lesões no Brasil: efeitos, avanços alcançados e desafios futuros. Lancet 2011; 6736(11):75-89.

4. Andrade-Barbosa TL, Xavier-Gomes LM, Barbosa VA, Caldeira AP. Mortalidade masculina por causas externas em Minas Gerais, Brasil. Cien Saude Colet 2013; 18(3):711-719.

5. Gadelha CAG, Costa L. Integração de fronteiras: a saúde no contexto de uma política nacional de desenvolvimento. Cad Saude Publica 2007; 23(Supl. 2):S214-S226.

6. Costa FR, Rocha MM. Nova dinâmica dos municípios do Paraná. Mercator 2014; 13(2):91-104.

7. Albuquerque JLC. A dinâmica das fronteiras: deslocamento e circulação dos "brasiguaios" entre os limites nacionais. Horizontes Antropol 2009; 15(31):137-166.

8. Gordis L. Measuring the occurrence of disease: II. Mortality. In: Gordis L, editor. Epidemiology. $3^{\text {a }}$ ed. Philadelphia: Elsevier Saunders; 2004. p. 48-70.

9. Latorre MRDO, Cardoso MRA. Análise de séries temporais em epidemiologia: uma introdução sobre os aspectos metodológicos. Rev Bras Epidemiol 2001; 4(3):145-152.

10. Lozada EMK, Mathias TAF, Andrade SM, Aidar T. Tendência da mortalidade por homicídios no Estado do Paraná, segundo Regionais de Saúde, 1979 a 2005. Rev Bras Epidemiol 2009; 12(2):258-269.

11. Salla F, Alvarez MC, Oi AH. Homicídios na Faixa de Fronteira do Brasil: 2000-2007 [documento na Internet]. Relatório de Pesquisa do Projeto Violência e Fronteiras FAPESP/CNPq. São Paulo: Núcleo de Estudos da Violência da USP (NEV/USP); 2011 [acessado 21 Nov 2017]. Disponível em: http://nevusp.org/ blog/2011/01/03/homicdios-na-faixa-de-fronteira-do -brasil-2000-2007/

12. Giovanella L, Guimarães L, Nogueira VMR, Lobato LVC, Damacena GN. Saúde nas fronteiras: acesso e demandas de estrangeiros e brasileiros não residentes ao SUS nas cidades de fronteira com países do MERCOSUL na perspectiva dos secretários municipais de saúde. Cad Saúde Pública 2007; 23(Supl. 2):S251-S266.

13. Malta DC, Minayo MCS, Soares Filho AM, Silva MMA, Montenegro MMS, Ladeira RM, Morais Neto OL, Melo AP, Mooney M, Naghavi M. Mortalidade e anos de vida perdidos por violências interpessoais e autoprovocadas no Brasil e Estados: análise das estimativas do Estudo Carga Global de Doença, 1990 e 2015. Rev Bras Epidemiol 2017; 20(Supl. 1):142-156.

14. Dávila-Cervantes CA, Pardo-Montaño AN. Magnitud y tendencia de la mortalidad por homicidios en Colombia y México, 2000-2011. Rev Panam Salud Publica $2014 ; 36(1): 10-16$.
15. Barreto MS, Teston EF, Latorre MRDO, Mathias TAF, Marcon SS. Mortalidade por acidentes de trânsito e homicídios em Curitiba, Paraná, 1996-2011. Epidemiol Serv Saúde 2016; 25(1):95-104.

16. Alves WA, Correia DS, Barbosa LLB, Lopes LM, Melânia MIASM. Violência letal em Maceió-Al: estudo descritivo sobre homicídios, 2007-2012. Epidemiol Serv Saúde 2014; 23(4):731-740.

17. Otzen T, Sanhueza A, Manterola C, Hetz M, Melnik T. Homicide in Chile: trends 2000-2012. BMC Psychiatry 2015; 15(312):1-5.

18. Chaparro-Narváez P, Cotes-Cantillo K, León-Quevedo W, Castañeda-Orjuela C. Mortalidad por homicidios en Colombia, 1998-2012. Biomédica 2016; 36(4):572-582.

19. Matzopoulos RG, Thompson ML, Myers JE. Firearm and nonfirearm homicide in 5 South African cities: a retrospective population-based study. Am J Public Health $2014 ; 104(3): 455-460$.

20. von-Doellinger V, Campos M, Mendes L, Schramm J. The 2008 Global Burden of Disease study in Brazil: a new methodological approach for estimation of injury morbidity. Rev Panam Salud Publica 2014; 36(6):368-375.

21. López-López MV, Pastor-Durango MP, Giraldo-Giraldo CA, García-García HI. Delimitación de fronteras como estrategia de control social: el caso de la violencia homicida en Medellín, Colombia. Salud Colect 2014; 10(3):397-406.

22. Groenewald P, Neethling I, Evans J, Azevedo V, Naledi T, Matzopoulos R, Nannan N, Daniels J, Bradshaw D. Mortality trends in the City of Cape Town between 2001 and 2013: reducing inequities in health. $S$ Afr Med J 2017; 107(12):1091-1098.

23. Amaral LBM, Vasconcelos TB, Sá FE, Silva ASR, Macena RHM. Violência doméstica e a Lei Maria da Penha: perfil das agressões sofridas por mulheres abrigadas em unidade social de proteção. Rev Estud Fem 2016; 24(2):521-540.

24. Rocha GG, Nunes BP, Silva EF, Wehrmeister FC. Análise temporal da mortalidade por homicídios e acidentes de trânsito em Foz do Iguaçu, 2000-2010. Epidemiol Serv Saude 2016; 25(2):323-330.

25. Costa FAMM, Trindade RFC, Santos CB. Mortes por homicídios: série histórica. Rev Latino-Am Enfermagem 2014; 22(6):1017-1025.

26. Ruotti C, Almeida JF, Regina FL, Massa VC, Peres MFT. A vulnerabilidade dos jovens à morte violenta: um estudo de caso no contexto dos "Crimes de Maio". Saude Soc 2014; 23(3):733-748.

27. Ribeiro AP, Souza ER, Sousa CAM. Lesões provocadas por armas de fogo atendidas em serviços de urgência e emergência brasileiros. Cien Saude Colet 2017; 22(9):2851-2860.

28. Scorzafave LG, Soares MK, Dorigan TA. Vale a pena pagar para desarmar? Uma avaliação do impacto da campanha de entrega voluntária de armas sobre as mortes com armas de fogo. Estud Econ 2015; 45(3):475-497. 
29. Lucena SEF, Moraes RM. Detecção de agrupamentos espaço-temporais para identificação de áreas de risco de homicídios por arma branca em João Pessoa, PB. Bol Cien Geod 2012; 18(4):623-605.

30. Morais RM, Costa AL. Uma avaliação do Sistema de Informações sobre Mortalidade. Saúde Debate 2017; 41(esp.):101-117.

Artigo apresentado em 30/07/2018

Aprovado em 05/11/2018

Versão final apresentada em 07/11/2018 